\title{
Assessment of Sorafenib and AntiVEGF Combination Therapy Response which Added to Neoadjuvant Therapy in two Pediatric Metastatic Ewing Sarcoma Patients by Fluorine-18 Fluorodeoxyglucose Positron Emission Tomography (18F-PET) Method: It may Determine the Prognosis
}

Nurdan Tacyildiz ${ }^{{ }^{*}}$, Gulsah Tanyildiz ${ }^{1}$, Cigdem Soydal ${ }^{2}$, Elgin Ozkan ${ }^{2}$, Ozlem Kucuk ${ }^{2}$, Gulsan Yavuz $^{1}$, Emel Unal ${ }^{1}$, Handan Dincaslan $^{1}$, Yusuf Yildiz $^{3}$, Esra Pekpak $^{1}$, Zulfikar Gordu ${ }^{1}$ and Basak Aksoy ${ }^{1}$

${ }^{1}$ Department of Pediatric Hematology-Oncology, Ankara University, School Of Medicine, Ankara, Turkey

${ }^{2}$ Department of Nuclear Medicine, Ankara University, School Of Medicine, Ankara, Turkey

${ }^{3}$ Department of Orthopedics, Ankara University, School Of Medicine, Ankara, Turkey

"Corresponding author: Nurdan Tacyildiz, Department of Pediatric Hematology-Oncology, Ankara University, School Of Medicine, Ankara- 06100, Turkey, Tel: 905422353424; E-mail: nurdantacyildiz@yahoo.com

Received date: Dec 29, 2014, Accepted date: Feb 04, 2015, Publication date: Feb 09, 2015

Copyright: @ 2015 Tacyildiz N, et al. This is an open-access article distributed under the terms of the Creative Commons Attribution License, which permits unrestricted use, distribution, and reproduction in any medium, provided the original author and source are credited.

\begin{abstract}
Background: The prognosis is still poor in metastatic Ewing sarcoma (ES) patients. We aimed to assess importance of 18F-PET/CT in 2 pediatric local invasive/ metastatic Ewing sarcoma patients which have been performed antiVEGF and Sorafenib besides their neo-adjuvant and adjuvant chemotherapies (ChT) to improve their prognosis. Response to therapy has been followed by $18 \mathrm{~F}-\mathrm{PET} / \mathrm{CT}$ in addition to MR imaging.

Patients: First patient was a 12 year-old-girl who was suffering from right leg pain and diagnosed with Ewing sarcoma (ES). 18F-PET/CT showed a locally invasive mass with $14 \times 5 \mathrm{~cm}$ dimensions located on one third of proximal tibia with a 5,1 SUVmax value was observed. After 4 cycles of conventional ChT beside antiVEGFSorafenib, SUVmax: 2,1 and tumor necrosis was $80 \%$. Patient received local Radiotherapy (RT) and still in remission after 18 months. Second patient was a 13 year-old-girl who admitted with swelling and pain on right scapula. MR imaging revealed a mass causing deterioration on right scapula and surrounding soft tissue. Diagnosed as ES. PET/CT showed that: primary lesion SUV max 12,4 value, beside another metastatic lesion on left fifth rib with 3,4 SUVmax,confirmed by MRI and biopsi. In addition to conventional neo-adjuvant ChT regimen for ES, antiVEGF and sorafenib were added. There was no 18F-PET/CT involvement during pre-surgical evaluation. Tumor necrosis was $\% 98$ and surgical border was tumor negative. Postoperative adjuvant chemotherapy with antiVEGF and sorafenib continued untill end of the treatment protocol. Besides, local RT. Primary region of the tumor and metastatic lesion were still 18F-PET/CT imaging negative, by the enf of the therapy. Patient is still well after 15 months.
\end{abstract}

Conclusion: 18F-PET/CT imaging can predict tumor response to neoadjuvant/adjuvant ChT which includes promising antiVEGF and sorafenib therapies in pediatric ES patients in the manner of the primary tumor necrosis ratio and metastatic evaluations.

Keywords: Pediatric metastatic Ewing sarcoma; Neoadjuvant/ adjuvant chemotherapy; Sorafenib; AntiVEGF; 18F-PET/CT

\section{Introduction}

Prognosis of metastatic Ewing sarcoma is still unsatisfied. Recently, in order to improve prognosis, sorafenib (a multikinase inhibitor) and anti-VEGF (anti-angiogenic drug) therapy have been added to the classic neoadjuvant therapy in our clinic. We aimed to emphasize the importance of (18)F-fluorodeoxyglucose (FDG) uptaken by Positron Emission Tomography/Computed Tomography (PET/CT) imaging in two pediatric patients diagnosed as Ewing sarcoma and also we were able to follow primary and metastatic disease status and neo-adjuvant therapy response to AntiVEGF and sorafenib.

\section{Case 1}

A 12 year old girl complained of right upper leg pain and swelling since 3 months admitted to orthopedy clinic. On her physical examination there was a difference in diameter between the two low extremities. On X-ray evaluation, there was an onion skin like periost reaction which arise from proximal to distal part of right femur, accompanied with a non-deplaced fracture line was really remarkable. On laboratorial assesstment; blood count, serum LDH (140-304 IU/L) and ALP (0-500 U/L) level was normal and also there was not any positive viral serologic result. MR image of mass revealed the cystic and necrotic components of the tumor with $14 \times 12 \mathrm{~cm}$ diameter and biopsy was performed for pathological diagnosis. It was not seen a skip metastasis near by the primary region on MR imaging. Patient diagnosed with Ewing sarcoma (ES) and referred to our oncology department for chemotherapy. When PET/CT imaging was assessed, it was seen that there was a pathological involvement (SUV max:9.8) 
Citation: Tacyildiz N, Tanyildiz G, Soydal C, Ozkan E, Kucuk O, et al. (2015) Assessment of Sorafenib and AntiVEGF Combination Therapy Response which Added to Neoadjuvant Therapy in two Pediatric Metastatic Ewing Sarcoma Patients by Fluorine-18 Fluorodeoxyglucose Positron Emission Tomography (18F-PET) Method: It may Determine the Prognosis. J Nucl Med Radiat Ther 6: 212. doi:10.4172/2155-9619.1000212

Page 2 of 3

which was belong to primary region spread around the soft tissue and accompanied with right external iliac lymph node metastasis (SUV $\max : 4.2)$ (Figure 1).

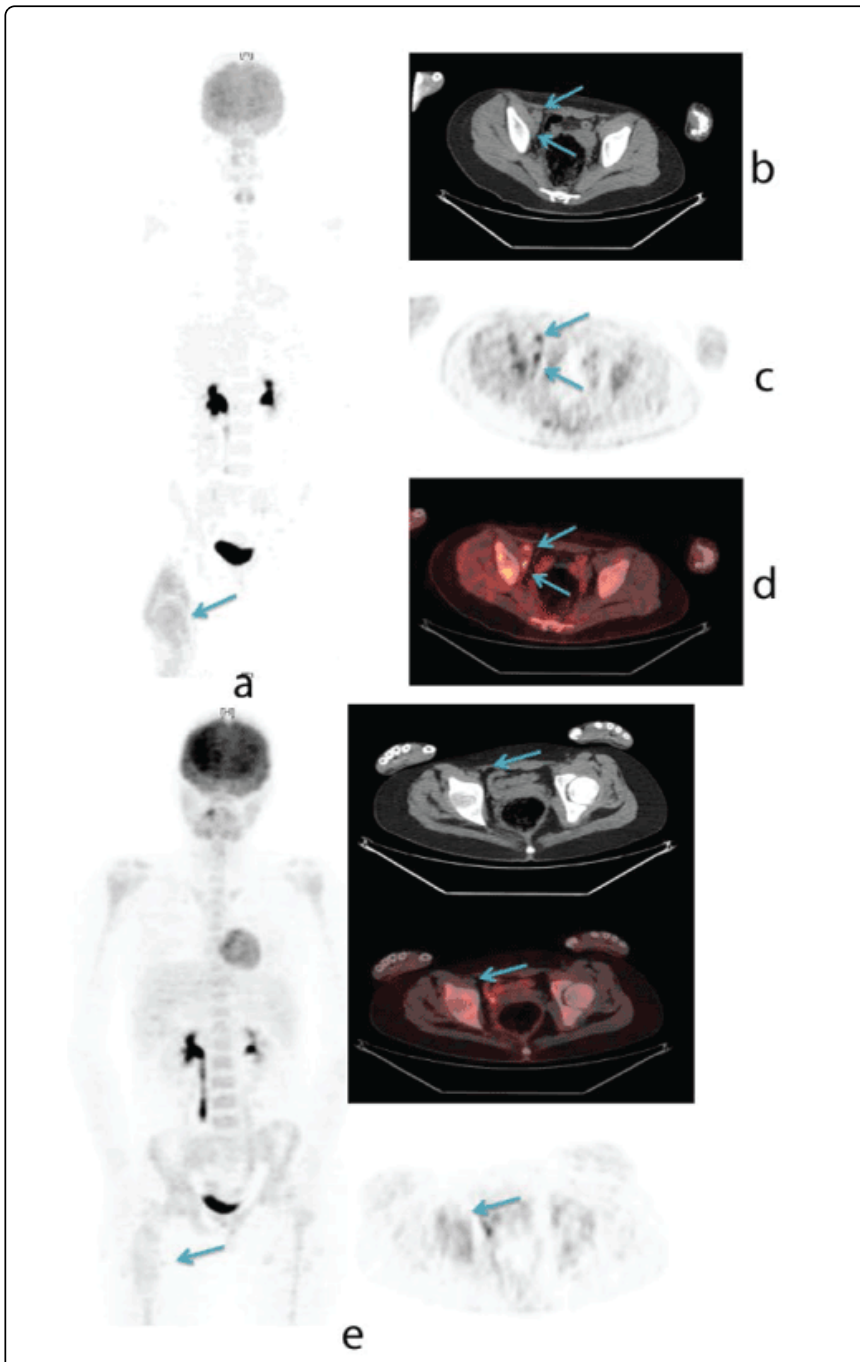

Figure 1: In pretreatment maximum intensity projection (a), axial CT (b), PET (c) and Fused (d) images intense 18F-FDG uptake (SUVmax: 9.8) was seen in primary tumor located in the proximal femur. Additionally pathological uptake was detected in right external iliac lymph nodes. (SUVmax:4.2). After treatment dramatical decrease in SUV of primary tumor (SUVmax: 3.7) and (e) absence of uptake in external iliac lymph nodes was confirmed.

At the time of diagnosis, disease was staged as metastatic/infiltratif because of environmental soft tissue and external iliac lymph node invasion. Inhibitors of tyrosine kinase and antiangiogenic agents were also added to conventional neo-adjuvant chemotherapy protocol concomitantly. Anti VEGF therapy was started as $10 \mathrm{mg} / \mathrm{kg} / \mathrm{dosage}$ for every two weeks intravenously and sorafenib ( an oral form of tyrosine kinase inhibitör) was started as $2 \times 200 \mathrm{mg}$ /day between the two chemotherapy cycles regularly. At the end of four chemotherapy cycles, dimension of mass $(28 \times 17 \mathrm{~mm})$ and value of SUVmax (3.7) were regressed. Metastatic external iliac lymp node involvement was completely disappeared (Figure 2). Malignant tumor resection and reconstruction with allograft were performed as primary regional surgery. The percentage of necrosis was $\% 95$ and microscopic surgery borders were tumor negative. Conventional adjuvant chemotherapy besides anti VEGF and sorafenib were continued until the end of therapy and 5040 cGy RT was performed regionally. During the postoperative follow up no residual disease or recurrence was detected at MRI and PET/CT imaging showed complete improvement of the pathological involvement. Anti VEGF and sorafenib treatments were continued regularly for 6 months after completion of conventional therapy. Patient is still on remission after 18 months of follow up period.

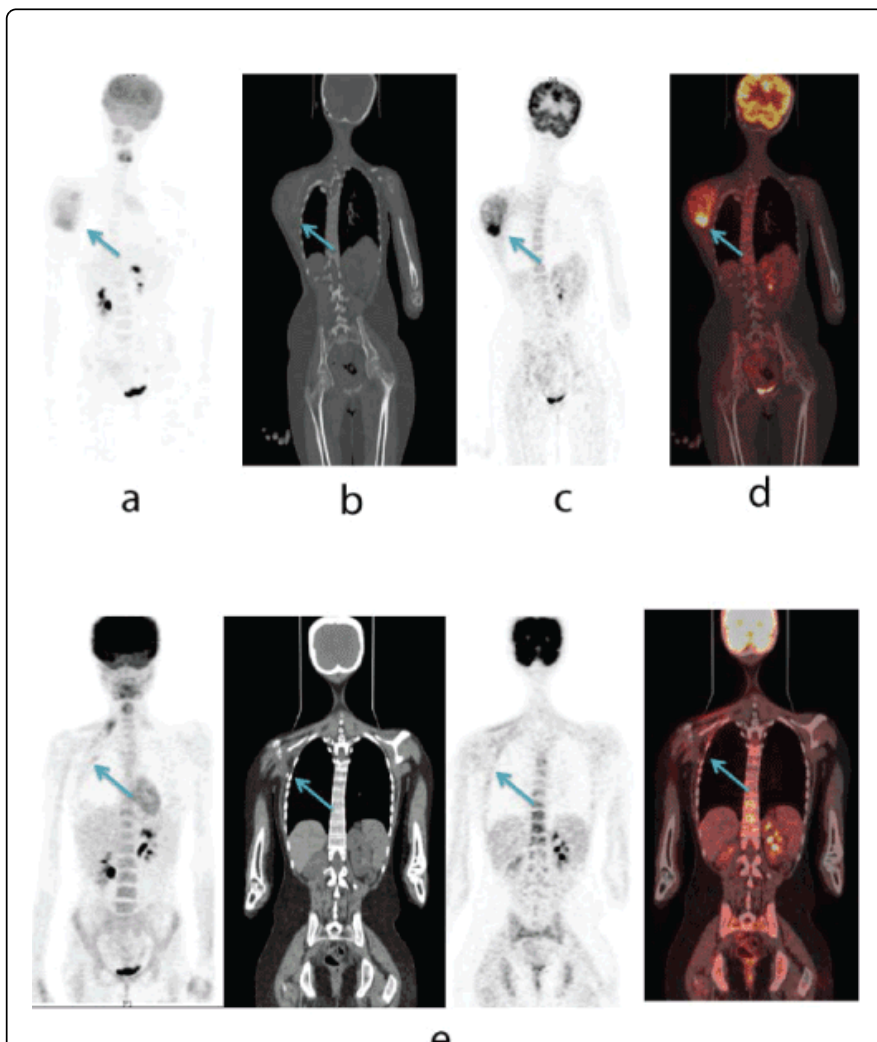

Figure 2: Nonhomogenous pathological uptake in primary scapular tumor (SUVmax: 12.4) was shown by maximum intensity projection (a), coronal CT (b), PET (c) and Fused (d) images of pretreatment 18F-FDG PET/CT. (e) This uptake was disappeared after compliation of treatment.

\section{Case 2}

A 13 year old girl with swelling and pain on right scapula admitted to orthopedy clinic. Patient's symptoms had started 4 months ago. There was a painful mass sized $20 \times 18 \mathrm{~cm}$ diameter on the rigt shoulder during physical examination. MR imaging revealed a mass $(10 \times 6.5 \mathrm{~cm})$ causing deterioration on right scapula and having large components of soft tissue involvement. When the pathological diagnosis was consistent with ES, patient was referred to our oncology clinic. On laboratoral assessment, sedimentation rate (ESR) was $130 \mathrm{~mm} /$ hour (0-20 mm/hour) and LDH: $430 \mathrm{U} / \mathrm{L}$ (140-304) level was abnormally increased where as ALP level was normal. Viral serology studies have not show positivity of any virus. PET/CT study was performed and 
Citation: Tacyildiz N, Tanyildiz G, Soydal C, Ozkan E, Kucuk O, et al. (2015) Assessment of Sorafenib and AntiVEGF Combination Therapy Response which Added to Neoadjuvant Therapy in two Pediatric Metastatic Ewing Sarcoma Patients by Fluorine-18 Fluorodeoxyglucose Positron Emission Tomography (18F-PET) Method: It may Determine the Prognosis. J Nucl Med Radiat Ther 6: 212. doi:10.4172/2155-9619.1000212

Page 3 of 3

primary lesion with SUV max 12.4 value had been detected and confirmed by MRI and biopsy. Patient has been accepted as locally metastatic ES. In addition to conventional neo-adjuvant chemotherapy regimen for ES, antiVEGF and sorafenib were added. Clinical and radiological features of the mass were regressed with no PET involvement during pre-surgical evaluation. The percentage of necrosis was $\% 98$ and surgical border was tumor negative. Postoperative adjuvant chemotherapy with antiVEGF and sorafenib continued until end of the treatment protocol. Besides, 5040 cGy RT was performed regionally. Primary region of the tumor was still PET/CT imaging negative, by the enf of the therapy which has been confirmed by MRI either. Patient is still on follow up for 15 months without any disease after therapy.

\section{Discussion}

Presence of metastasis at the time of diagnosis is the most important prognostic factor for bone tumors. Another well known reality about the surveillance of metastatic bone tumors are not convincing enough. It is clear that new treatment modalities are required [1-4]. Anti VEGF is a monoclonal antibody treatment as targeted therapy for inhibition of new tumor vessels formation and when combined with tyrosine kinase inhibitors they potentiate each others. In adults it is used commonly in metastatic and refractory solid tumors (breast, colorectal, renal and hepatocellular) [5]. In recent years in addition to conventional neoadjuvant chemotherapy, multikinase inhibitors such as sorafenib, Anti VEGF and antiangiogenic agents are also given in order to improve the prognosis $[6,7]$. In our report we aimed to present two pediatric patients with regional and distant metastatic Ewing sarcoma at the time of diagnosis, treated with multikinase inhibitors and anti VEGF agents in addition to conventional neoadjuvant chemotherapy and followed by $\mathrm{PET} / \mathrm{CT}$ for monitoring their response to therapy. We observed that initiation of early combination treatment with targeted agents in metastatic disease improved the results of surveillance and treatment success and we were able to show the correlation between tumor necrosis ratio and PET/CT SUV max value. Conventionally, serials of CT and/or MRI studies are performed after radiotherapy and/or chemotherapy for determining the changes of tumor sizes. However, regression of tumor size by treatment needs relatively long time beside necrotic and/or fibrous tissue forming after treatment limits the expected regression of tumor size. In that manner, PET/CT is more sensitive to predict tumor necrosis than other imaging studies that mainly shows tumor size and lokal involvement of the tumor. Therefore, normalization of FDG uptake after radiotherapy and chemotherapy is an evidence of good response to treatment. Relatively poor impairment of FDG involvement indicates incomplete treatment respond. There is a positive correlation between treatment success and FDG involvement in many malignancies [8-10].

In our first case, primary tumor side SUV max was 3.7 after 4 cycle of treatment although it was 9,8 at the beginning which shows good response beside tumor regression from $14 \mathrm{~cm}$ diameter to $28 \mathrm{~mm}$. After surgery, tumor necrosis ratio was $\% 95$, which was well correlated with pre surgical PET/CT evaluation. We also aimed to emphasize the role of 18F-PET/CT imaging on response of metastatic regions to treatment which includes antiVEGF and sorafenib. External iliac lymp node SUV max was negative after treatment, although it was 4.2 at the beginning. In our second case, SUV max was completely regressed from 12.4 to 0 with treatment at the locally metastatic primary lesion and MRI results very good correlated with PET/CT (mass disappeared totally).

In conclussion; Acording to our preliminary results as shown here in two pediatric patients, $18 \mathrm{~F}-\mathrm{PET} / \mathrm{CT}$ imaging is able to predict primary or metastatic tumor response to neoadjuvant therapy which includes new targeted therapies like antiVEGF and sorafenib in pediatric Ewing sarcoma patients.

\section{References}

1. Nesbit ME Jr, Gehan EA, Burgert EO Jr, Vietti TJ, Cangir A, et al. (1990) Multimodal therapy for the management of primary, nonmetastatic Ewing's sarcoma of bone: a long-term follow-up of the First Intergroup study. J Clin Oncol 8: 1664-1674.

2. Cotterill SJ, Ahrens S, Paulussen M, Jürgens HF, Voûte PA, et al. (2000) Prognostic factors in Ewing's tumor of bone: analysis of 975 patients from the European Intergroup Cooperative Ewing's Sarcoma Study Group. J Clin Oncol 18: 3108-3114.

3. Paulussen M, Ahrens S, Burdach S, Craft A, Dockhorn-Dworniczak B, et al. (1998) Primary metastatic (stage IV) Ewing tumor: survival analysis of 171 patients from the EICESS studies. European Intergroup Cooperative Ewing Sarcoma Studies. Ann Oncol 9: 275-281.

4. Shankar AG, Pinkerton CR, Atra A, Ashley S, Lewis I, et al. (1999) Local therapy and other factors influencing site of relapse in patients with localised Ewing's sarcomUnited Kingdom Children's Cancer Study Group (UKCCSG). Eur J Cancer 35: 1698-1704.

5. Dalal S, Berry AM, Cullinane CJ, Mangham DC, Grimer R, et al. (2005) Vascular endothelial growth factor: a therapeutic target for tumors of the Ewing's sarcoma family. Clin Cancer Res 11: 2364-2378.

6. Costelloe CM, Chuang HH, Madewell JE (2014) FDG PET/CT of primary bone tumors. AJR Am J Roentgenol 202: 521-531.

7. Gupta K, Pawaskar A, Basu S, Rajan MG, Asopa RV, et al (2011) Potential role of FDG PET imaging in predicting metastatic potential and assessment of therapeutic response to neoadjuvant chemotherapy in Ewing sarcoma family of tumors. Clin Nucl Med 36: 973-977.

8. Hebert ME, Lowe VJ, Hoffman JM, Patz EF, Anscher MS (1996) PET in the pretreatment evaluation and follow- up of NSCLC patients treated with radiotherapy. Am J Clin Oncol 19: 416-421.

9. Love C, Din AS, Tomas MB Kalapparambath TP, Palestro CJ (2003) Radionuclide bone imaging: an illustrative review. RadioGarphics 23: 341-358.

10. Peller PJ (2013) Role of positron emission tomography/computed tomography in bone malignancies. Radiol Clin North Am 51: 845-864. 